\title{
Early Aggressive Therapy for Patients with Juvenile Idiopathic Arthritis: Are We There Yet?
}
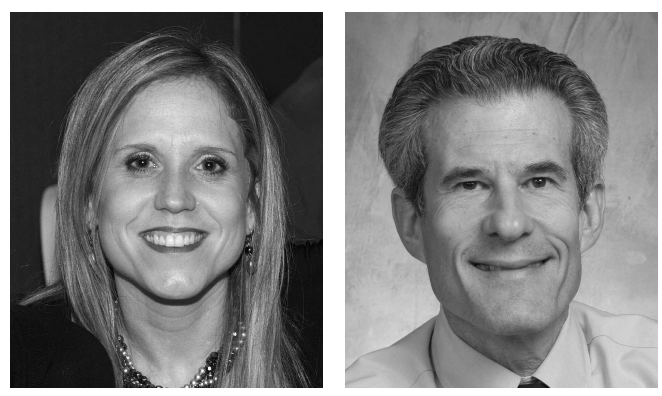

This is an extremely exciting time in the field of pediatric rheumatology. Advances in understanding the pathogenesis of juvenile idiopathic arthritis (JIA) have led to novel treatments with resultant marked improvements in patient outcomes, as is eloquently summarized in a review by Lovell and colleagues ${ }^{1}$.

Critical to the study of these new therapies has been the development of standardized outcome measures. These are not only relevant to research studies but also assist in making treatment decisions in clinical practice. The definition of improvement by the American College of Rheumatology (ACR) core set response criteria ${ }^{2}$ and more recently, criteria of inactive disease and remission, both on and off medication, have been developed and adopted by the $\mathrm{ACR}^{3}$ (Table 1). Application of these standardized outcome measures has greatly facilitated the reporting and generalizing of study results, as well as allowed comparison of results between trials.

Earlier studies, in the "prebiologics era," used differing measures for reporting remission and classifying patients; and the length of followup varied. As a result, it was extremely difficult to compare results between studies. A Canadian multicenter retrospective cohort study reported that 10 years following diagnosis of juvenile rheumatoid arthritis $^{4}$, the probability of remission (defined as 2 years without taking medication) was $37 \%$ in patients with systemic disease, $47 \%$ in oligoarthritis, $23 \%$ in rheumatoid factor (RF)-negative polyarthritis, and $6 \%$ in RF-positive polyarthritis ${ }^{5}$. This is in contrast to a multicenter retrospective cohort study (Italy and the United States) in which, after a median of 7.7 years post-JIA diagnosis (similar categories considered as above), $40 \%$ of patients were in remission (defined as 6 months without taking medication). However, at 2 years without medications (the more rigid criteria defined by Oen, $e t \mathrm{al}^{5}$ ), only $28 \%$ of patients were in remission ${ }^{6}$. Overall, in cohort studies reported in the prebiologic era, patients had poor outcomes with little chance of remission, particularly patients with more severe polyarthritis.

Outcome studies reported more recently in the biologics era, using the ACR definitions for clinical inactive disease (CID) and clinical remission while not taking medication (CR), report significantly better outcomes. Ringold, et $a l^{7}$ reported a retrospective polyarticular JIA cohort study (28\% RF-positive, 104 children) of patients followed for a median of 27.4 months (range 6-77). Eighty percent of patients achieved CID at a median time of 7.6 months after initiation of treatment; $37 \%$ received anti-tumor necrosis factor (TNF) therapy, $11.5 \%$ within their first 6 months of care. Forty-seven percent achieved clinical remission while taking medication, none achieved CR, and 20\% never achieved CID. The investigators of the ReACCh-Out study (Canadian Research in Arthritis in Canadian Children emphasizing Outcomes) report on the prospective inception cohort of newly diagnosed patients $(<6$ mos $)$ with all subtypes of JIA. Of the data from 1104 patients, the probabilities of attaining an active joint count of 0 exceeded $70 \%$ within 2 years in all JIA categories, except for RF-positive polyarthritis $(48 \%)$. The probability of attaining CR (completely without medications for 12 months and no active disease) within 5 years was $46-57 \%$ across JIA categories except for patients with polyarthritis $(0 \%$ RF-positive, 14\% RF-negative). The probability of starting anti-TNF was $11.1 \%$ in the RF-positive polyarthritis patients within the first 6 months of care ${ }^{8}$.

There is no debate regarding the efficacy of anti-TNF- $\alpha$ therapy for patients with polyarticular JIA. A recent review reports increasing use over the last decade ${ }^{9}$. The optimal timing of introduction and withdrawal of these medications is not known. Unique in its design, the Trial of Early Aggressive Therapy (TREAT) in JIA studied the effect of early introduction of etanercept (ETN) plus prednisolone (PSL) and methotrexate (MTX) compared to MTX alone on the attainment of CID at 6 months ${ }^{10}$. Eighty-five patients

See Extension trial of early aggressive therapy in JIA, page 2459

Personal non-commercial use only. The Journal of Rheumatology Copyright @ 2014 . All rights reserved. 
Table 1. ACR definitions of clinical inactive disease and clinical remission for JIA. From: Wallace, et al. Arthritis Care Res 2011;63:929-3633 with permission.

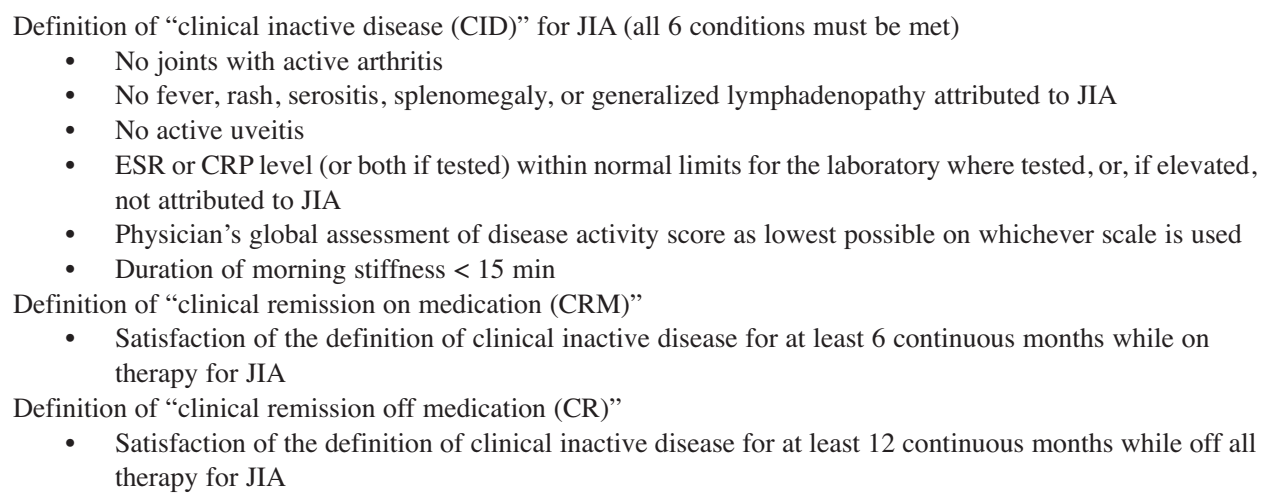

ACR: American College of Rheumatology; JIA: juvenile rheumatoid arthritis; ESR: erythrocyte sedimentation rate; CRP: C-reactive protein.

with polyarticular JIA $<12$ months' duration were studied in this prospective, randomized, double-blind, placebo-controlled trial.

Patients received either MTX $0.5 \mathrm{mg} / \mathrm{kg} /$ week (maximum $40 \mathrm{mg}$ ) subcutaneously (SC), ETN $0.8 \mathrm{mg} / \mathrm{kg} /$ week (maximum $50 \mathrm{mg}$ ) SC, and PSL $0.5 \mathrm{mg} / \mathrm{kg} /$ day (maximum $60 \mathrm{mg}$ ) orally and tapered to $0 \mathrm{mg}$ by 17 weeks (arm 1); or MTX (same dosage as arm 1), ETN placebo, and PSL placebo (arm 2). The study design limited the time for the patient taking placebo; if subjects failed to meet an intermediate endpoint of $70 \%$ improvement according to ACR Pediatric criteria at 4 months, they entered the open-label ETN/MTX/PSL arm. By 6 months, CID had been achieved in 17 of 42 patients $(40 \%)$ in arm 1 and 10 of 43 patients $(23 \%)$ in arm 2 (chi-squared $=2.91, \mathrm{p}=0.088$ ).

After 12 months, clinical remission while taking medication (CRM) was achieved in 9 patients in arm 1 and 3 patients in arm $2(p=0.053)$. The trial failed to meet its primary endpoint (attainment of CID at $6 \mathrm{mos}$ ); the authors note this was potentially explained by the overly ambitious goal of $60 \%$ CID in the MTX/ETN/PSL arm as well as higher (potentially more effective) doses of MTX used. Importantly, CID was induced in 32\% of patients by 6 months and in $66 \%$ by 12 months in this severely affected group of patients. The TREAT study participants were the most severely affected patients with polyarthritis, compared to the patients reported by Ringold, et $a l^{7}$ and Guzman, et $a l^{8}$, and this difference explains the lower proportion of TREAT patients attaining CID and CRM.

In this issue of The Journal, Wallace, et al report on an open-label extension phase of the TREAT trial ${ }^{11}$. Data from $76 \%(48 / 63)$ of eligible patients were reported, with a mean observation period of 39 months (range 24-54): Fifty percent of patients spent more than half their followup in CID; $88 \%$ of patients achieved CID at one or more study visits, $54 \%$ achieved CRM, but $12 \%$ never achieved CID. Forty-three of 48 patients in the extension study were receiving MTX and ETN and only 5 were taking MTX alone. Interestingly, baseline active joint counts and duration of symptoms prior to treatment did not correlate with proportion of time in CID. This is in contrast to the original 6-month trial that reported an OR for achieving CID of 1.324 for each month earlier that a patient was treated ${ }^{10}$.

One of the main concerns with the TREAT trial design is the use of prednisone $(0.5 \mathrm{mg} / \mathrm{kg} /$ day to a maximum of 60 $\mathrm{mg} /$ day) with ETN and MTX. The study did not address the potential effect on outcomes of early prednisone use (tapered off by 4 mos). A provocative editorial in The Journal earlier this year argued that even short-term use of steroids in pediatric patients is unacceptable and can have side effects such as infection and affect self-image, growth, metabolism, and bone structure ${ }^{12}$.

Despite the growing body of literature on longterm safety in the use of biologics, the risks of potential serious longterm side effects (e.g., malignancy) are relatively unknown $^{13,14,15,16,17}$, and certainly in the short term, serious infections are possible ${ }^{18}$.

The TREAT extension study reported intermediate 2-year outcomes on a severely affected group of patients with aggressively treated polyarticular JIA with superior outcomes.

Early aggressive therapy was similarly evaluated in newly diagnosed patients with polyarticular JIA in the ACUTE-JIA trial reported by Tynjala, et $a l^{19}$. This was a 54-week open-label trial of 60 patients randomized to 1 of 3 treatment arms: infliximab plus MTX (TNF); MTX, sulfasalazine, and hydroxychloroquine (COMBO), or MTX (MTX) alone. The primary endpoint was $75 \%$ improvement according to the ACR Pediatric criteria, achieved in $100 \%$ receiving TNF, $65 \%$ in the COMBO group $(95 \% \mathrm{CI}$

Personal non-commercial use only. The Journal of Rheumatology Copyright @ 2014 . All rights reserved. 
44-86\%), and 50\% taking MTX (95\% CI 28-72\%), p < 0.0001 . CID according to the Wallace criteria ${ }^{3}$ (applied in a posthoc analysis) was achieved in 13 patients (68\%) receiving TNF (95\% CI 47-89\%); 7 (35\%) in the COMBO group (95\% CI 6-44\%); and 5 (20\%) taking MTX (95\% CI $3-39 \%) ; \mathrm{p}=0.001$. Similarly to the TREAT extension trial, patients taking TNF spent a mean of 25 weeks (46\%; 95\% CI 17-33) in CID, significantly longer than those in the COMBO group (11 weeks; 95\% CI 5-17), or those taking MTX (5 weeks; 95\% CI 1-9); $\mathrm{p}=0.003$.

Now that anti-TNF agents have been shown to be effective in JIA, investigators are turning to the question of how long patients must be maintained on this treatment. Considerations include the potential for short-term and longterm side effects from anti-TNF agents, and in many parts of the world, the difficulty accessing these drugs. Ideally, results from translational research will guide the safe and rational use of these biologics, with defined recommendations on the duration of treatment once a patient has achieved CRM. A recent exploratory study by Ringold, et al to identify biomarkers of disease activity may help to answer this question. In their study of 31 patients with polyarticular course JIA, serum amyloid A, C-reactive protein, interleukin 6 , and matrix metalloproteinase 3 were elevated in the active disease group as compared to the children with CID and controls ${ }^{20}$. Similarly, using pretreatment blood samples from the TREAT trial, a panel of immunologic assays (immune signatures including phenotypical and functional data) could predict CID vs no CID after 6 months of the MTX/ETN/PSL aggressive treatment arm with $90 \%$ sensitivity and specificity ${ }^{21}$.

Despite the superior outcomes reported, early universal introduction of anti-TNF agents for all patients with polyarticular JIA is not currently feasible; nor has there been, in our opinion, sufficient longterm data to support the safety profile of this approach. Access to these therapies is an issue; and their cost is considerable. Certainly, in the Canadian landscape, with both private and publicly funded insurers, the use of biologic agents is not yet a first-line treatment. In addition, there is a marked variability in access to biologics between provinces ${ }^{22}$.

The priority for investigators is to identify patients most in need of anti-TNF therapy (based on their genetic, immunologic, and/or biomarker susceptibility) in whom early introduction will result in an alteration of the longterm prognosis in terms of sustained CRM. In addition, studies of the potential of biologic agents to modify radiographic evidence of disease progression are lacking. The very early introduction of biologic therapies should be targeted to patients at highest risk of poor functional or radiographic outcome to mitigate risk of potential adverse effects and limit cost. Careful clinical observation, and data collection and analysis, will be critical to understanding which patients should receive early biologic treatment, as well as how early treatment should be started.

ROBERTA A. BERARD, MD, MSc, FRCPC, Pediatric Rheumatologist, Children's Hospital,

London Health Sciences Centre; Assistant Professor of Pediatrics, Western University;

RONALD M. LAXER, MD, FRCPC, Professor of Pediatrics and Medicine, University of Toronto, Staff Rheumatologist, The Hospital for Sick Children, Toronto, Ontario, Canada.

Address reprint requests to Dr. Laxer; E-mail: ronald.laxer@sickkids.ca

\section{REFERENCES}

1. Lovell DJ, Ruperto N, Giannini EH, Martini A. Advances from clinical trials in juvenile idiopathic arthritis. Nat Rev Rheumatol 2013;9:557-63.

2. Giannini EH, Ruperto N, Ravelli A, Lovell DJ, Felson DT, Martini A. Preliminary definition of improvement in juvenile arthritis. Arthritis Rheum 1997;40:1202-9.

3. Wallace CA, Giannini EH, Huang B, Itert L, Ruperto N; Childhood Arthritis Rheumatology Research Alliance, et al. American College of Rheumatology provisional criteria for defining clinical inactive disease in select categories of juvenile idiopathic arthritis. Arthritis Care Res 2011;63:929-36.

4. Brewer EJ, Bass J, Baum J, Cassidy J, Fink C, Jacobs J. Current proposed revision of JRA criteria. Arthritis Rheum 1977;20 Suppl:195-9.

5. Oen K, Malleson PN, Cabral DA, Rosenberg AM, Petty RE, Cheang M. Disease course and outcome of juvenile rheumatoid arthritis in a multicenter cohort. J Rheumatol 2002;29:1989-99.

6. Wallace CA, Huang B, Bandeira M, Ravelli A, Giannini EH. Patterns of clinical remission in select categories of juvenile idiopathic arthritis. Arthritis Rheum 2005;52:3554-62.

7. Ringold S, Seidel KD, Koepsell TD, Wallace CA. Inactive disease in polyarticular juvenile idiopathic arthritis: current patterns and associations. Rheumatology 2009;48:972-7.

8. Guzman J, Oen K, Tucker LB, Huber AM, Shiff N, Boire G, et al. The outcomes of juvenile idiopathic arthritis in children managed with contemporary treatments: results from the ReACCh-Out cohort. Ann Rheum Dis 2014 May 19 (E-pub ahead of print).

9. Mannion ML, Xie F, Curtis JR, Beukelman T. Recent trends in medication usage for the treatment of juvenile idiopathic arthritis and the influence of tumor necrosis factor inhibitors. J Rheumatol 2014;41:2078-84

10. Wallace CA, Giannini EH, Spalding SJ, Hashkes PJ, O’Neil KM, Zeft AS, et al. Trial of early aggressive therapy in polyarticular juvenile idiopathic arthritis. Arthritis Rheum 2012;64:2012-21.

11. Wallace CA, Ringold S, Bohnsack J, Spalding SJ, Brunner HI, Milojevic D, et al. Extension study of participants from the TRial of Early Aggressive Therapy in Juvenile Idiopathic Arthritis. J Rheumatol 2014;41:2459-65.

12. Lehman TJ. The TREAT study - trial or tribulation? J Rheumatol 2014;41:1038-9.

13. Hashkes PJ, Uziel Y, Laxer RM. The safety profile of biologic therapies for juvenile idiopathic arthritis. Nat Rev Rheumatol 2010;6:561-71.

14. Bernatsky S, Rosenberg AM, Oen KG, Duffy CM,

Personal non-commercial use only. The Journal of Rheumatology Copyright (C) 2014. All rights reserved 
Ramsey-Goldman R, Labrecque J, et al. Malignancies in juvenile idiopathic arthritis: a preliminary report. J Rheumatol 2011; 38:760-3.

15. Diak P, Siegel J, La Grenade L, Choi L, Lemery S, McMahon A. Tumor necrosis factor alpha blockers and malignancy in children: forty-eight cases reported to the Food and Drug Administration. Arthritis Rheum 2010;62:2517-24.

16. Hasija RP, Silverman ED, Cho S, Fung L, Benseler SM, Cameron B, et al. A170: Neoplasms in pediatric patients with rheumatic diseases exposed to biologics - A quarternary centre's experience [abstract]. Arthritis Rheumatol 2014;66 Suppl 3:S220.

17. Ruperto N, Martini A. Juvenile idiopathic arthritis and malignancy. Rheumatology 2014;53:968-74.

18. Beukelman T, Xie F, Baddley JW, Chen L, Delzell E, Grijalva CG, et al. Brief report: incidence of selected opportunistic infections among children with juvenile idiopathic arthritis. Arthritis Rheum 2013;65:1384-9.
19. Tynjala P, Vahasalo P, Tarkiainen M, Kröger L, Aalto K, Malin M, et al. Aggressive combination drug therapy in very early polyarticular juvenile idiopathic arthritis (ACUTE-JIA): a multicentre randomised open-label clinical trial. Ann Rheum Dis 2011;70:1605-12.

20. Ringold S, Lu L, Wallace CA, Sasso EH, Eastman PS. Initial assessment of multi-biomarker disease activity assay in JIA [abstract]. Arthritis Rheumatol 2014;66 Suppl 3:S10.

21. Rossetti M, Spreafico R, Zhang H, Moshref M, Singer NG, Lovell DJ, et al. Predictive markers of therapeutical outcome and their role in the ethiopathology of juvenile idiopathic arthritis [abstract]. Arthritis Rheum 2012;64 Suppl 10:S1097.

22. Leblanc CM, Lang B, Bencivenga A, Chetaille AL, Dancey P, Dent $\mathrm{P}$, et al. Access to biologic therapies in Canada for children with juvenile idiopathic arthritis. J Rheumatol 2012;39:1875-9.

J Rheumatol 2014;41:2343-6; doi:10.3899/jrheum.141051 